\title{
Research on the Evolution Mechanism of Urban Distribution System's Resource Integration
}

\author{
Chen Liang ${ }^{1}$, Dong $\mathrm{Mu}^{2}$ and ZhengqiangJiang ${ }^{3}$ \\ ${ }^{1,2}$ Beijing Jiaotong University, Beijing 100044, China; \\ ${ }^{1,3}$ Beijing Wuzi University, Beijing 101149, China \\ liangchen727@sina.com, ${ }^{2}$ mueast@163.com, ${ }^{3}$ injt19891011@126.com
}

\begin{abstract}
Urban distribution system, as an important component of urban logistics, plays a vital role in urban economic development and its residents' living standards improving. This paper explored the evolution mechanism of urban distribution system's resource integration evolution by using self-organization theories; it concluded the condition evolution mechanism, dynamic mechanism as well as its principle mechanism at first. After that, it constructed the evolution model of urban distribution system's resource integration evolution process respectively under the condition of single order parameter and two order parameters. At the end of this paper, it summed up the "four stages, three phase transitions" characteristics of urban distribution system's resource integration evolution process.
\end{abstract}

Keywords: urban distribution system, resource integration, self-organization theory, evolution mechanism

\section{Introductions}

Urban distribution system (UDS) plays a crucial role in promoting urban economic development, improving urban competitiveness, as well as achieving a sustainable development of urban economy and environment. As we can see, UDS has already been one of the key factors in urban developing and even the implementation of national strategy, especially under the current strategy of seeking context of urbanization. After the launching of "logistics industry restructuring and revitalization plan" in 2009, establishing a sound UDS to improve urban environment, ease urban traffic pressure, improve urban logistics operation efficiency and some other issues has been written into many local governments' work schedules. The government, enterprises, civil societies and other social subjects have contributed lots of positive forces to developing the UDS, and it has comes to some big success. But it is not optimistic enough when compared with developed countries, so establish a good urban distribution system comes to be particularly urgent.

This paper explored the UDS from resource perspective by using self-organization theories; it concluded the condition mechanism, dynamic mechanism as well as its principle mechanism at the beginning. And then, in order to get a deeper understanding of this issue, it constructed the evolution model of UDS' resource integration process respectively under the condition of single order parameter and two order parameters. It summed up the "four stages, three phase transition" characteristic at the end of this paper. 


\section{The Condition Mechanism of UDS' Resource Integration Evolution}

In the urban environment, the resource integration evolution process follows the principle of nonlinear interaction, and it forms an open system which was far away from equilibrium. The system evaluates to order state from disorder state, evaluates to high order state from low order state by exchange material and energy with external environment system, it forms a new system structures and new organization forms by inner self-organization, which comes to be a typical dissipative structures and the UDS' resource integration just carried out by this way $[1,2]$.

The interaction forms between distribution resources are nonlinear, and the resource input from the government and enterprises are also varied. The typical distribution resource, such as vehicles, facilities, financial, human resource and so on, effect obvious differently under different distribution demand and resource combinations. Meanwhile, different resource combinations will break original UDS, which would change the UDS' original state of the base entropy $[3,4]$.

The root reason, which lead to the unbalanced of UDS, is the unevenly distributed of material resource and energy. As we can analyze, the input categories and quantities of each distribution organization, such as capital, technology, equipment, manpower and some others, are varied. But the deeper reason as we can analyze, is the varied of organization size, organization efficiency, organizational structure, management ability, market conditions and some other factors.

The above analysis shows that UDS is an open system which was far away from equilibrium, and under the nonlinear interaction of internal and external aspects of its subsystem and its corresponding elements, the system develops from disordered state to ordered state, or develops from primary ordered state to a higher ordered state continuously and spontaneously. So as we can conclude, UDS' resource integration evolution processes have the following 4 conditions.

1) An open system architecture, it is one of the most important prerequisite of urban distribution system's resource integration.

2) Faraway from equilibrium state, it is the most important reason which lead urban distribution system's resource from chaos, scattered to orderly integration.

3) Random fluctuations, it is the most important internal reason induce urban distribution system's resource integration.

4) Nonlinear function, it is the fundamental mechanism of urban distribution system's resource integration.

\section{The Dynamic Mechanism of UDS' Resource Integration Evolution}

\subsection{Driving Force within the System}

Driving force within the system refers to the dynamic factor which drives the UDS' resource integration process within the system; it is the foundation and root of UDS' resource integration evolution. The main driving force within the system include the following three aspects, which are the interests conflict between resource subjects, resource subjects' autocratic and its uneven capacity.

1) The interests conflict between resource subjects

The distribution resource subjects can be divided into two categories, which are government and enterprises, the enterprises are more closely associated with the market. As we can analyze 
from industry chain perspective, any complete distribution business involved varied different distribution subjects, it needs to integrate logistics, information, capital and other resource to meet the end customer's distribution demand. In this process, due to the resource owners profit-driven, it leads to a lot of problems, such as information distortion, poor timeless, poor resource matching and so on, all these problems have big negative effects on resource subjects' decision, and it will cause contradictions and conflicts between resource subjects.

2) Resource integration subjects' autocratic

The final driving force of UDS' resource integration evolution comes from industry, commerce, consumer, emergency and other areas' distribution demand. Similar with pulling production model, the distribution integration is also goal-driven; the subjects of distribution resource have the power to decide the direction of integration evolution directly or indirectly. These subjects' product characteristics, demand patterns, demand scale, demand level and other characteristics decide the way of resource integration by professional distribution organization directly. Thus, a more powerful resource subject, who requires excessive servicer standards, lower distribute prices, or looser constraints on the partners, will decrease the system's performance, destroy the synergy.

3) The unbalance capacity of system subjects

In microscopic distribution business, the ultimate performance depends on the whole distribute process, includes its order delivery, procurement stocking, delivery warehousing, sorting, handling, distribution processing, packaging, transportation and many other activities. This process may involve multiple companies together, and as the unbalance capacity of different system subjects, the entire system's operation maybe turbulent, and the disorder of the whole system will increase.

\subsection{The External Influence of the System}

The external influence of the system mainly includes the continue change of its demand, the competition of other areas, the government's support behavior and technical innovation of this area.

1) The continues change of its demand

The initial distribution system need to adapt to urban's economic and social developing. Agile manufacturing, JIT, mass customization and other new production models are making new demand of distribution system constantly. Meanwhile, the changes of leading industrial structure, urban space, and urban functional areas and so on have a significant impact on its distribution system. If the distribution system cannot deal with these positive entropy felicitously, the existing system will be disintegration.

2) The competition comes from other areas

As the application of new technology and equipment, the system's speed and its price advantage will be disappear. As we know, the enhancement of Postal courier's service content and its capabilities, it predatory UDS' personnel, capital and other resource, so it affects the stability of its UDS and makes UDS developing from disorderly state to orderly state, primary orderly to higher orderly.

3) The government's support behavior 
As we know, rational planning for UDS' development from government level can guide and promote urban distribution optimal allocation its resource effectively. Government regulation and planning can overcome the defects that the market itself cannot handle.

4) Technical innovation

Fully exert the force of distribution system to environment and promote science or technology in UDS can help create a more extensive distribution requirement, which can help increase the matching between market demand and its distribution systems.

\section{The Principle Mechanism of UDS' Resource Integration Evolution}

\subsection{Path Dependence Theory}

Path dependence is a term that has come into common use in both economics and society. In all instances that path dependence is asserted, the assertion amounts to some version of "history matters." Path dependence can mean just that: Where we are today is a result of what has happened in the past. For example, the statement "we saved and invested last year and therefore we have assets today" might be more fashionably expressed as, "the capital stock is path dependent"[5-7]. It was first introduced into social science research by American economic historian David PA [8]. However, North DC explains the evolution of institutional economics successfully by "path dependency" in 1981 and won the Nobel Prize of Economics in 1993 by these studies, which make "path dependency" theory of fame widely known [9]. In recent years, "path dependence" has a lot of application in the interpretation of industrial organization and industrial organizations. For example, Antonelli analyzed the "path dependence phenomenon" in industrial organization [10], Martin R., etc., investigated the "path dependence phenomenon" in regional economic evolution [11], and Bellaiche studied the "path dependence phenomenon in economic growth.

UDS' integration belongs to economic evolution area, the "path dependence" phenomenon manifested in the process that the benefits incurred in the integration process is greater than the cost incurred, and the UDS' resource would have been self-organization integration until the self-organization integration benefits equals to the self-organization integration costs.

\subsection{Emergence Theory}

Another important feature of UDS' resource integration is the emergence theory. The emergence phenomenon refers to the attributes and behavior patterns that the whole system have, but its consolidation doesn't have, which also can be described as "the whole is greater than the sum of its parts" or "1 $+1>2$ ". The emergence phenomenon of UDS is generally expiated by the integration process, and it always reflects in scale and structure effects [13-15]. To this issue, theorists have a wealth research literature, and we don't discuss it here anymore. 


\section{The Model Construction and Analysis}

\subsection{The Model of UDS' Resource Integration Evolution with the Condition of Single Order Parameter}

In the process of UDS' resource integration evolution, the first and most obvious change is the inventories of UDS' resource. Therefore, we choose this parameter in the model of UDS' resource integration process with the condition of single order parameter.

In the case of single order parameter, the phase transition situations of this system can be described by this single order parameter and the equation can be described as formula (1) which can be seen as below:

$$
\frac{\mathrm{dX}}{\mathrm{dt}}=-\frac{\mathrm{dS}(\mathrm{X})}{\mathrm{dX}}
$$

The self-organization model with the condition of single order parameter can be described as follows:

$$
\frac{\mathrm{dX}}{\mathrm{dt}}=-\gamma * \mathrm{X}+\left(\alpha * \mathrm{X}+\rho * \mathrm{X}^{3}\right)=-(\alpha-\gamma) * \mathrm{X}+\rho \mathrm{X}^{3}
$$

So the potential function can be described as follows:

$$
S(X)=-\int \frac{d X}{d t} d X=\int\left[(\alpha-\gamma) * X-\rho X^{3}\right] d X=\frac{1}{2}(\alpha-\gamma) X^{2}-\frac{1}{4} \rho X^{4}
$$

In formula (3):

$\mathrm{X}$ refers to the order parameter, it is a function of time $\mathrm{t}$;

$\alpha$ refers to the influence coefficient of signal order parameter's self-organization evolution;

$\gamma$ regers to the damping coefficient $(\gamma>0)$;

$\rho$ refers to the attenuation coefficient of order parameter $\mathrm{X}(\mathrm{t})(\rho>0)$.

(1) If $\alpha \leq \gamma$ :

As $\frac{d S(X)}{d X}=-\frac{d X}{d t}=0$, so we can caculate that $X=0$, while $\left.\frac{d}{d X}\left(\frac{d X}{d t}\right)\right|_{X=0}>0$. Thus, there isn't any phase transition point.

The system determined by single order parameter $\mathrm{X}$ is the simplest, continuous expansion system, this kind of system can be exist in theory, but we haven't found such a system in reality.

(2) If $\alpha>\gamma$ :

As $\frac{\mathrm{dS}(\mathrm{X})}{\mathrm{dX}}=-\frac{\mathrm{dX}}{\mathrm{dt}}=0$, so we can caculate that $\mathrm{X}_{1}=-\sqrt{\frac{(\alpha-\gamma)}{\rho}}, \mathrm{X}_{2}=0 ; \mathrm{X}_{3}=\sqrt{\frac{(\alpha-\gamma)}{\rho}}$, 由 $\frac{\mathrm{d}}{\mathrm{dX}}\left[\frac{\mathrm{dS}(\mathrm{X})}{\mathrm{dX}}\right]=\frac{\mathrm{d}}{\mathrm{dX}}\left[-\frac{\mathrm{dX}}{\mathrm{dt}}\right]=(\alpha-\gamma)-3 \rho X^{2}=0$, and thus $\mathrm{X}_{1}^{\prime}=-\sqrt{\frac{(\alpha-\gamma)}{3 \rho}}, \mathrm{X}_{2}^{\prime}=\sqrt{\frac{(\alpha-\gamma)}{3 \rho}}$.

Thus, there are three phase transition points, and these evaluations can be divided into 4 stages, and the corresponding transition points can be valued $\mathrm{X}_{1}, \mathrm{X}_{2}, \mathrm{X}_{3}$, and the 4 stages are $\left(-\infty, X_{1}\right),\left(X_{1}, X_{2}\right),\left(X_{2}, X_{3}\right)$ and $\left(X_{3},+\infty\right)$ respectively.

The self-organization evolution of UDS' resource integration evolution process can be seen in Table 1. 
Table 1. The Self-organization Evolution Process with the Condition of Single Order Parameter

\begin{tabular}{|c|c|c|c|c|}
\hline Stages & Stage 1 & Stage 2 & Stage 3 & Stage 4 \\
\hline $\mathbf{X}$ & $\left(-\infty, \mathrm{X}_{1}\right)$ & $\left(\mathrm{X}_{1}, \mathrm{X}_{2}\right)$ & $\left(\mathrm{X}_{2}, \mathrm{X}_{3}\right)$ & $\left(\mathrm{X}_{3},+\infty\right)$ \\
\hline $\mathbf{T}$ & $\left(0, \mathrm{t}_{1}\right)$ & $\left(\mathrm{t}_{1}, \mathrm{t}_{2}\right)$ & $\left(\mathrm{t}_{2}, \mathrm{t}_{3}\right)$ & $\left(\mathrm{t}_{3},+\infty\right)$ \\
\hline $\mathbf{S}(\mathbf{X})$ &,$+ \nearrow$ &,$+ \searrow$ &,$+ \nearrow$ &,$+ \searrow$ \\
\hline $\mathbf{S}(\mathbf{X})^{\prime}$ & - & + & + & - \\
\hline $\mathbf{S}(\mathbf{X})^{2}$ & $-\nearrow$ & $-\nearrow+$ & $+\searrow$ & $+\searrow-$ \\
\hline $\mathbf{S}(\mathbf{X})^{3}$ & - & - & + & + \\
\hline
\end{tabular}

Factor $T$ can be calculated by formula (2).

\subsection{The Model of UDS' Resource Integration Evolution with the Condition of Two Order Parameters}

In the process of UDS' resource integration evolution, the first and most obvious change is the inventories of UDS' resource, and followed by this is the change of UDS' resource efficiency. Therefore, we choose the inventories of UDS' resource and its efficiency as the parameters in the model of UDS' resource integration evolution with the condition of two order parameters.

$$
\left\{\begin{array}{c}
\frac{\mathrm{dS}(\mathrm{X})}{\mathrm{dt}}=\alpha \mathrm{S}+\alpha_{1} \mathrm{X}_{1}+\alpha_{2} \mathrm{X}_{2}+\alpha_{3} \mathrm{X}_{1} \mathrm{X}_{2}+\mathrm{F}(\mathrm{t}) \\
\frac{\mathrm{dX_{1 }}}{\mathrm{dt}}=\left(\alpha_{4}-\gamma_{1}\right) \mathrm{X}_{1}-\rho \mathrm{X}_{1}^{2}-\beta_{1} \mathrm{X}_{1} \mathrm{X}_{2} \\
\frac{\mathrm{dX}_{2}}{\mathrm{dt}}=-\gamma_{2} \mathrm{X}_{2}+\beta_{2} \mathrm{X}_{1}^{2}
\end{array}\right.
$$

In this case, there are positive feedback and negative feedback interactions between these activities and order parameters, but the potential function can't explain the interaction of these dual order parameters. Therefore, this paper constructed the model of UDS' resource integration process with the condition of two order parameters by Langev in formula.

In formula (4):

$\mathrm{X}_{1}, \mathrm{X}_{2}$ refers to the order parameters, and they are the function of time $t$, which can be expressed as $X_{1}(t)$ and $X_{2}(t)$;

$\mathrm{S}(\mathrm{X})$ refers to the state parameter, and it is the function of $\mathrm{X}_{1}(\mathrm{t})$ and $\mathrm{X}_{2}(\mathrm{t})$;

$\alpha$ refers to the natural feedback coefficient in the system integration process;

$\alpha_{1}$ refers to the influence parameter of $X_{1}$ to the system integration evolution;

$\alpha_{2}$ refers to the influence parameter of $X_{2}$ to the system integration evolution;

$\alpha_{3}$ refers to the influence parameter of $X_{1}$ to $X_{2}$;

$\mathrm{F}(\mathrm{t})$ refers to the random fluctuation phenomena in its integration process;

$\alpha_{4}$ refers to the gain factor ot these two order parameters;

$\gamma_{1}$ refers to the damping of $\mathrm{X}_{1}$;

$\gamma_{2}$ refers to the damping of $\mathrm{X}_{2}$;

$\rho$ refers to the attenuation coefficient of order parameter $X_{1}$;

$\beta_{1}$ refers to the force coefficient between these 2 order parameters;

$\beta_{2}$ refers to the correlation coefficient of these 2 order parameters;

$\mathrm{t}$ refers to time factor.

When $\frac{d S(X)}{d t}=\frac{d X_{1}}{d t}=\frac{d X_{2}}{d t}=0$, the system comes to be stable. In this situation, we can 
construct the linear matrix equation of $\mathrm{S}(\mathrm{X}), \mathrm{X}_{1}$ and $\mathrm{X}_{2}$, which can be seen in formula (5) as below.

$$
\left\{\begin{aligned}
\frac{\mathrm{dS}(\mathrm{X})}{\mathrm{dt}} & =\alpha \mathrm{S}+\alpha_{1} \mathrm{X}_{1}+\alpha_{2} \mathrm{X}_{2}+\alpha_{3} \mathrm{X}_{1} \mathrm{X}_{2}=0 \\
\frac{\mathrm{dX_{1 }}}{\mathrm{dt}} & =\left(\alpha_{4}-\gamma_{1}\right) \mathrm{X}_{1}-\rho \mathrm{X}_{1}^{2}-\beta_{1} \mathrm{X}_{1} \mathrm{X}_{2}=0 \\
\frac{\mathrm{dX}}{\mathrm{dt}} & =-\gamma_{2} \mathrm{X}_{2}+\beta_{2} \mathrm{X}_{1}^{2} \quad=0
\end{aligned}\right.
$$

Thus, we can calculate:

$$
\left[\begin{array}{ccc}
\alpha & \alpha_{1}+\frac{1}{2} * \alpha_{3} \mathrm{X}_{2} & \alpha_{2}+\frac{1}{2} * \alpha_{3} \mathrm{X}_{2} \\
0 & \alpha_{4}-\gamma_{1}-\rho \mathrm{X}_{1}-\frac{1}{2} * \beta_{1} \mathrm{X}_{2} & -\frac{1}{2} * \beta_{1} \mathrm{X}_{1} \\
0 & \beta_{2} \mathrm{X}_{1} & -\gamma_{2}
\end{array}\right] *\left[\begin{array}{c}
\mathrm{S} \\
\mathrm{X}_{1} \\
\mathrm{X}_{2}
\end{array}\right]=\left[\begin{array}{l}
0 \\
0 \\
0
\end{array}\right]
$$

Formula (6) means that:

$$
\left(\lambda_{1}-\alpha\right) *\left[\lambda_{2}-\left(\alpha_{4}-\gamma_{1}\right)\right]^{*}\left(\lambda_{3}+\gamma_{2}\right)=0
$$

Thus, we can calculate the eigenvalues $\lambda_{1}=\alpha, \lambda_{2}=\alpha_{4}-\gamma_{1}$ and $\lambda_{3}=-\gamma_{2}$, as $\gamma_{2}$ refers to the damping of $\mathrm{X}_{2}$ and $\lambda_{3}<0$, so the stability of UDS' resource integration process mainly depend by $\alpha, \alpha_{4}$ and $\gamma_{1}$.

(1)if $\alpha<0$ and $\alpha_{4}<\gamma_{1}$

In this case, the value of all eigenvalues are negative, and there is a unique equilibrium point $(0,0,0)$, the system evaluate curves converge to 0 , which means the 2 order parameters decrease with time factor $t$. At this time, the systematic random fluctuation phenomenon can't cause the evolution curve fluctuations, and the system comes to the state of disordered self-organization. The curve of these two order parameters' evaluation process and the state parameter of the system can be seen in Figure 1.

(2)if $\alpha<0$ and $\alpha_{4}=\gamma_{1}$

In this case, the system is in a critical state of phase change, the order parameter guide the system forward evaluating, and the system approaching constantly towards the phase transition point under random fluctuation force, but as the order parameter' force is less than the macroscopic evolution force, the system doesn't appear abrupt, the UDS' resource integration can't be form a new ordered structure.
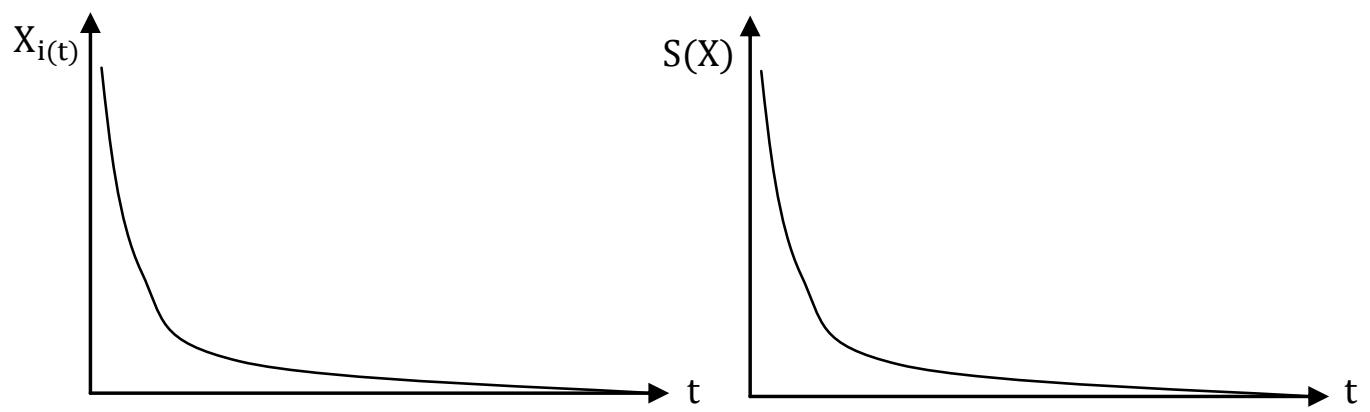

Figure 1. Evaluation Curves of Resource Integration and its Order Parameters when $\alpha<0$ and $\alpha_{4}<\gamma_{1}$ 


\section{(3)if $\alpha<0$ and $\alpha_{4}>\gamma_{1}$}

In this case, the system loses stability and consequent take phase transitions with order parameter changes, it formed a new primary ordered state gradually from disorder state, and it comes into a new stage of initial ordered. The system is disordered overall, and it is comes into locally ordered state. The curve of these two order parameters' evaluation process and the state parameter of the system can be seen in Figure 2.
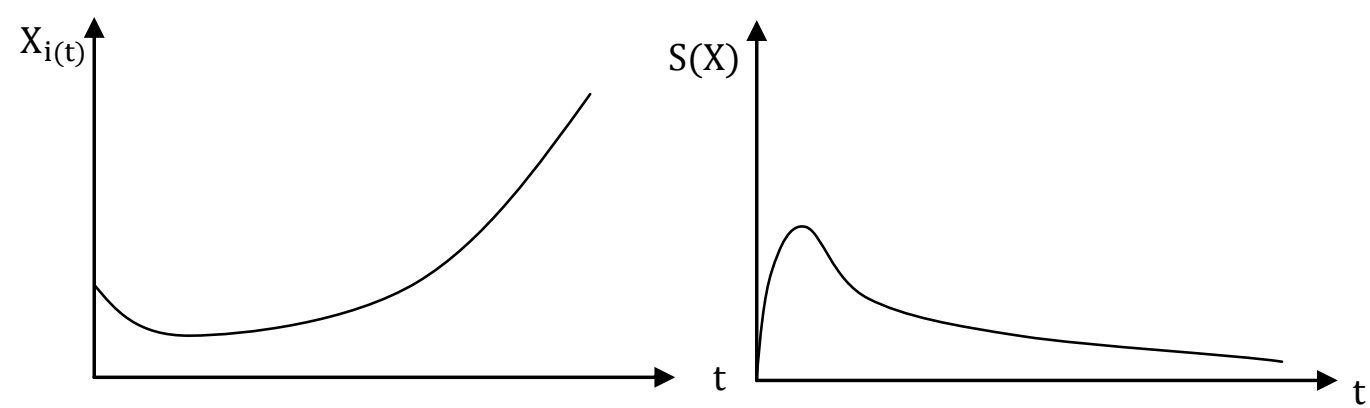

Figure 2. Evaluation Curves of Resource Integration and its Order Parameters when $\alpha<0$ and $\alpha_{4}>\gamma_{1}$

(4)if $\alpha=0$ and $\alpha_{4}=\gamma_{1}$

In this case, the system stays in a relatively stable state of equilibrium, and the UDS' resource integration comes to a higher of phase transition point, and the system comes to the next station as time goes on.

(5)if $\alpha>0$ and $\alpha_{4}<\gamma_{1}$

In this case, the system loses the steady state, and it will break through the stable critical point on the effect of the 2 order parameters. At this time, the system will exhibit more close structural characteristics, and the UDS' resource integration comes into self-organization. The curve of the two order parameters' evaluation process and the state parameter of the system can be seen in Figure 3.

(6) if $\alpha>0$ and $\alpha_{4}=\gamma_{1}$

In this case, the system comes into a relatively stable state of equilibrium, and it reaches a new, higher level of phase transition point.

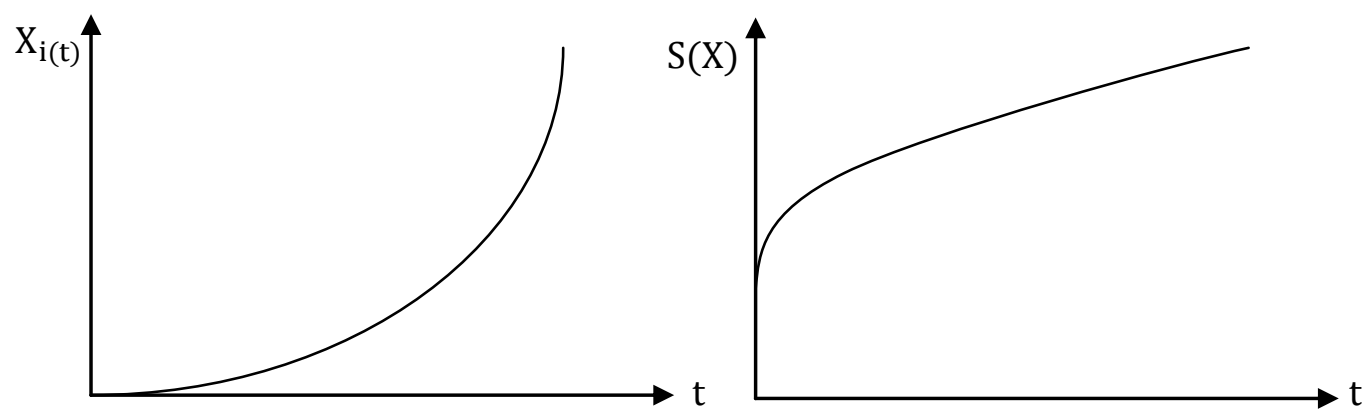

Figure 3. Evaluation Curves of Resource Integration and its Order Parameters when $\alpha \geq 0$ and $\alpha_{4}<\gamma_{1}$ 
(7)if $\alpha>0$ and $\alpha_{4}>\gamma_{1}$

In this case, the system loses the original stable structure by the force of order parameters, and the force of order parameters is greater than the force of macro evolution. So, small random fluctuations will cause significant structural changes, and the system will develop towards to a more stable structure, it will show a higher level of self-organization. The curve of the two order parameters' evolution process and the state parameter of the system can be seen in Figure 4.

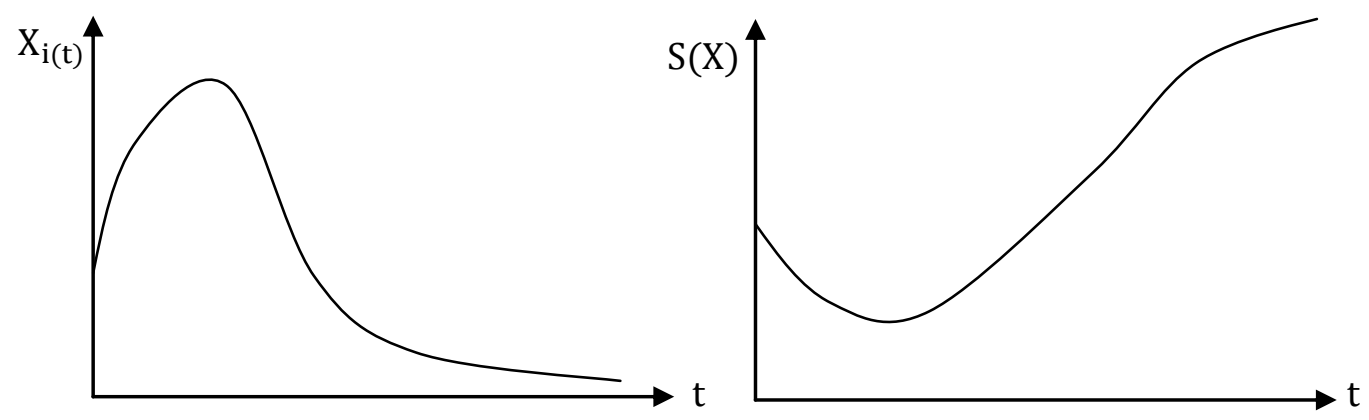

Figure 4. Evaluation Curves of Resource Integration and its Order Parameters when $\alpha \geq 0$ and $\alpha_{4}>Y_{1}$

After this stage, the two order parameters, which are the inventories of UDS' resource and its efficiency will be gradually withdraw, the system will researching new order parameter of the system

In summary, the evolution process with two order parameters is similar with the evolution process of signal order parameter, the system also comes to be "four stages, three phase change" phenomenon, and the characteristic of UDS' resource integration evolution can be seen in Table 2.

\section{Conclusion}

This paper explored the evolution mechanism of urban distribution system's resource integration evolution process by self-organization theories; it concluded the condition evolution mechanism, dynamic mechanism as well as its principle mechanism of this issue at the beginning. On the basis of these work, it constructed the evolution model and summed up the characteristics of urban distribution system's resource integration evolution respectively under the condition of single order parameter and two order parameters.

Table 2. The Self-Organization Evolution Process with the Condition of Two Order Parameters

\begin{tabular}{|c|c|c|c|c|}
\hline Coefficient & $\alpha<0$ & $\alpha<0$ & $\alpha<0$ & $\alpha=0$ \\
\hline values & $\alpha_{4}<\gamma_{1}$ & $\alpha_{4}=\gamma_{1}$ & $\alpha_{4}>\gamma_{1}$ & $\alpha_{4}=\gamma_{1}$ \\
\hline $\begin{array}{l}\text { Ordered } \\
\text { state }\end{array}$ & Disorder & \multirow{2}{*}{$\begin{array}{l}\text { Primary phase } \\
\text { transition point }\end{array}$} & $\begin{array}{l}\text { Overall disordered } \\
\text { and local order }\end{array}$ & \multirow{2}{*}{$\begin{array}{l}\text { Intermediate } \\
\text { phase } \\
\text { transition point }\end{array}$} \\
\hline $\begin{array}{c}\text { Organization } \\
\text { Status }\end{array}$ & $\begin{array}{l}\text { Independent } \\
\text { organization }\end{array}$ & & organization system & \\
\hline Coefficient & $\alpha>0$ & $\alpha>0$ & $\alpha>0$ & \\
\hline
\end{tabular}




\begin{tabular}{|c|c|c|c|}
\hline values & $\alpha_{4}<\gamma_{1}$ & $\alpha_{4}=\gamma_{1}$ & $\alpha_{4}>\gamma_{1}$ \\
\hline $\begin{array}{l}\text { Ordered } \\
\text { state }\end{array}$ & Primary Ordered & \multirow{2}{*}{$\begin{array}{c}\text { Advanced } \\
\text { phase transition } \\
\text { point }\end{array}$} & Advanced Ordered \\
\hline $\begin{array}{c}\text { Organization } \\
\text { Status }\end{array}$ & $\begin{array}{c}\text { Primary } \\
\text { self-organization }\end{array}$ & & $\begin{array}{c}\text { Advanced } \\
\text { self-organization }\end{array}$ \\
\hline
\end{tabular}

This paper first in-depth analysis the dissipative structure characteristic of UDS' resource integration, it analyzed the internal driving and external influence factors, such as economic environment, technology and policy environment. Later, it analyzed the principle mechanism of UDS' resource integration, which is "path dependence theory" and "emergence theory". Finally, it constructed the evolution model of urban distribution system's resource integration process respectively under the condition of single order parameter and two order parameters respectively, and analyzed the "four stages, three phase change" characteristic of UDS' resource integration process. Generally speaking, these papers strengthen the awareness of Research on the Evolution Mechanism of Urban Distribution System's Resource Integration.

\section{Acknowledgements}

The research work was supported by National Natural Science Foundation of China under Grant No. 71132008, Funding Project for Academic Human Resources Development in Institutions of Higher Learning under the Jurisdiction of Beijing Municipality and the Scientific Research Bases-Science and technology innovation platform of Beijing Wuzi University.

\section{References}

[1] Z. Ling-yun and M. Dong, "Research on Synergetic Evolution of Regional Logistics System", Journal of Beijing Jiaotong University (Social Sciences Edition), vol. 8, no. 2, (2009), pp. 26-28.

[2] X. Jie and J. Song-dong, "Establishment of the City Logistics Network System", China Business and Market, vol. 01, (2008).

[3] Y. Kai-yuan, "Analysis of Urban Allocation Guidance Transformation Principle”, Commercial research, vol. 03, (2011).

[4] Z. Fang, "Collaborative innovation impact on enterprise competitive advantage - Based on entropy theory and dissipative structure theory",Social scientists, vol. 08, (2011).

[5] L. Han-min, "Review on the theory of path dependence theory", Dynamic economics, vol. 6, (2003), pp. 65-69.

[6] S. J. Liebowitz and S. E. Margolis, "Path dependence, lock-in, and history", Journal of Law, Economics, \& Organization, (1995), pp. 205-226.

[7] Path dependence and creation[M]. Psychology Press, (2013).

[8] P. A. David, "Technical choice innovation and economic growth: essays on American and British experience in the nineteenth century", Cambridge University Press, (1975).

[9] Z. Guo-ping, "The nature view of Self organization”, Peking University Press, (1996).

[10] F. Xin, "The Research on the Path-dependence in the Process of the Evolution of Industry", Jinan University, (2006).

[11] C. Antonelli, "The economics of path-dependence in industrial organization", International Journal of Industrial Organization, vol. 15, no. 6, (1997), pp. 643-675.

[12] J. Meyer-Stamer, "Path dependence in regional development: persistence and change in three industrial clusters in Santa Catarina, Brazil", World development, vol. 26, no. 8, (1998), pp. 1495-1511.

[13] W. Jun, W. Lan-yi and L. Yi-jun, "CapabilityMaturityModelResearch of Third- party Logistics Enterprises", China Soft Science, vol. 11, no. 017, (2009).

[14] K. J. Hong, "The unintended consequences of the Helsinki Final Act: A path emergence theory perspective", International Political Science Review, vol. 34, no. 3, (2013), pp. 310-325.

[15] J. H. Turner, L. Beeghley and C. H. Powers, "The emergence of sociological theory", Sage, (2011). 

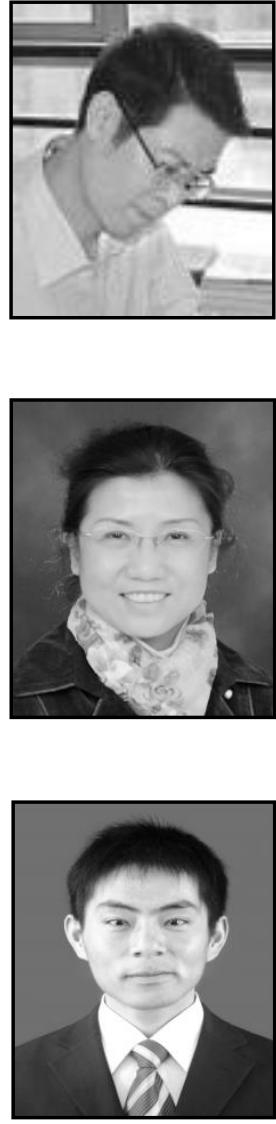

Chen Liang, He is afull instructor of Beijing Wuzi University (2005). $\mathrm{He}$ received his master degree in Management science and engineering from Beijing WuziUniversity, and now he is working on his $\mathrm{PhD}$ degree in Beijing Jiaotong University science 2009. His current research interests include different aspects of logistics engineering and Artificial Intelligence and Systems dynamics.

Dong Mu, She received her PhD degree in Business and Management School of TongjiUniversity (1999). Now she is a full time professor at Management School of Beijing Jiaotong University since 2005. Her current research interests include different aspects of logistics and supply system engineering, regional logistics and environmental evaluation and system dynamics.

Zhengqiang Jiang, he is a full master student of Beijing Wuzi University (2011). He received his undergraduate diploma in Huazhong University of Science and Technology, and now he is work on his master. His current research interests include different aspects of logistics engineering and Artificial Intelligence and Systems dynamics. 
International Journal of $\mathrm{u}$ - and e- Service, Science and Technology Vol.7, No.2 (2014) 